\title{
Decompaction of wet granular materials under freeze-thaw cycling
}

\author{
Maryam Pakpour, ${ }^{1,2,3, *}$ Nicolas Vandewalle, ${ }^{1}$ and Geoffroy Lumay ${ }^{1}$ \\ ${ }^{1}$ GRASP, Physics Department, University of Liège, B-4000 Liège, Belgium \\ ${ }^{2}$ Department of Physics, Institute for Advanced Studies in Basic Sciences (IASBS), Zanjan 45195-1159, Iran \\ ${ }^{3}$ Condensed Matter National Laboratory, Institute for Research in Fundamental Sciences (IPM), 19395-5531 Tehran, Iran
}

(Received 22 September 2017; revised manuscript received 20 October 2018; published 7 January 2019)

\begin{abstract}
The packing fraction dynamics of a wet granular material submitted to freeze-thaw cycling is investigated experimentally. The dynamics is strongly influenced by the liquid volume fraction $\omega$ in the considered range of $0.03<\omega<0.32$. This range of liquid contents covers different regimes of wetness from the creation of the capillary network until the formation of large clusters and finally close to the saturated case. For the liquid contents $\omega \gtrsim 0.15$, the pile experiences a decompaction until a particular value of the packing fraction 0.56 corresponding to a random loose packing configuration for monosized spheres. Moreover, the decompaction starts after a cycling number that decreases exponentially with the liquid content. Finally, we show that the packing dynamics can be well modeled on the basis of a Landau potential with an asymmetric double-well structure. The onset of decompaction represents the tendency of the system to stay in a metastable state. After several cycles, the forces induced by the thermal cycling and local stochastic rearrangements of the grains can drive the system to overcome the energy barrier of the cohesive forces.
\end{abstract}

DOI: 10.1103/PhysRevE.99.012901

\section{INTRODUCTION}

The packing dynamics of granular materials has received much attention since the precursor experiments of Bernal et al. [1] in the 1960s due to the ubiquitous applications from natural phenomena to technological processes. The main dimensionless parameter that characterizes the configuration of granular materials is the packing fraction $\eta$, which is defined as the ratio of the volume occupied by the grains to the volume of the assembly. The range of the random packing fraction obtained for monosized frictionless spheres is between the random loose packing $\eta_{\text {RLP }} \sim 0.55$ and the random close packing $\eta_{\mathrm{RCP}} \sim 0.64$. These values are significantly lower than the packing fraction $\eta_{\max } \sim 0.74$ corresponding to the highly ordered close-packed configuration that is obtained for hexagonal compact packing [2,3], although the precise values of the packing density depend on the geometry of particles, dispersity, and intergrain friction [4-6].

Granular materials are out-of-equilibrium systems exhibiting many complex behaviors such as jamming transition [7-9], size segregation [10,11], arch formation [12], and extremely slow compaction dynamics $[13,14]$. Most studies concerning granular media were focused on dry grains. However, it is well known that adding liquid strongly influences all mechanical behaviors of granular media, such as flow properties, instability, tensile strength, and agglomeration [15-19]. Liquid volume fraction $\omega$ in granular media is defined as the volume of added liquid divided by the total volume of the assembly. The distribution of the liquid inside a pile of granular beads has been measured by x-ray tomography images [20,21]. At very low liquid volume fraction, where

\footnotetext{
*mpakpour@iasbs.ac.ir
}

the transition from the dry to the wet state takes place, the fluid is trapped in the surface roughness of the particles. At higher liquid volume fraction in the so called Pendular states, a network of grains connected by liquid bridges is formed, which induces a cohesion due to the Laplace pressure. The optimum cohesion occurs for a critical liquid content of $\omega \sim 0.03$. Morphological studies performed by optical microscopy show that for the liquid content $\omega>0.03$ at the Funicular state, several liquid bridges merge into the aggregates. By increasing the liquid content situated at $\omega \sim 0.11$, most of the pores are filled with the liquid to form large contiguous wet clusters [22]. At this Capillary state, the Laplace pressure is reported to be still quite large. This is possibly due to the percolated liquid structure, which depends on the topology of the clusters $[22,23]$. Finally, the cohesive interaction in the system decreases to zero when the liquid content is around $\omega \sim 0.35$. So, when the granulate is completely saturated by the liquid, cohesion between the grains becomes negligible. Therefore, concerning the cohesiveness, the saturated configuration at rest is similar to the dry regime [19-24].

Despite the fact that the effect of cohesive force inside the wet granular materials is of fundamental interest, compaction dynamics of these systems is still an open problem [25]. Different driving methods have been considered to induce packing compaction, from tapping and shearing $[14,26,27]$ to flow pulses [28,29]. More recently, various studies examined the effect of thermal cycling on the grain packing [30-32]. Driving methods based on thermal cycling are particularly interesting in the wet case. Indeed, water has remarkable properties, such as a high surface tension, a maximum density at $4^{\circ} \mathrm{C}$, a high specific-heat capacity, a high heat of vaporization, and special behaviors during the phase transition. All of these properties serve as motivation to consider the role played by water in compaction dynamics of granular materials. Beyond 
the fundamental interest, several powerful phenomena in nature, such as ice-lens formation in soil $[33,34]$, the changing Arctic carbon cycle [35], landslides [36], and stone heave $[37,38]$, are induced by thermal cycling when the temperature drops below $0{ }^{\circ} \mathrm{C}$, for example on the frozen surface of Mars [39]. In this way, our results also may find a wider range of applications in several phenomena, from geophysics to the conservation of monuments. In the present study, we investigate experimentally the compaction dynamics of partially saturated granular materials due to thermal cycling. In this state, the dynamics is governed by the Laplace pressure inducing interaction between grains or clusters. The presence of liquid is found to lead to a variety of behaviors qualitatively different from partially saturated to fully saturated regimes. The packing dynamics of these systems is well modeled by the Landau potential with an asymmetric double-well structure.

\section{EXPERIMENTAL SETUP}

A sketch of the experimental setup is shown in Fig. 1. We used monodisperse spherical glass beads of diameter $d=500 \mu \mathrm{m}$ and density $2.5 \mathrm{~g} / \mathrm{cm}^{3}$ with additional water. The sample is confined into a borosilicate glass tube of internal diameter $D_{\text {tube }}=2.1 \mathrm{~cm}$. The diameter of the tube is large enough compared to the particle size to minimize the wall effects. Moreover, the tube walls were coated with a small amount of silicon oil in order to avoid sticking of the grains onto the walls. The wet granular materials were poured into the tube in small portions. To achieve the desired initial packing fraction $\eta_{0}$, the glass beads were compacted by vibrations in an ultrasonic cleaner tank with a $35 \mathrm{kHz}$ wave. This process had been carried out for all the samples with different water content. To submit the system to the heating and cooling thermal cycling, we placed the glass tube inside an isothermal box with dimensions $5 \times 5 \times 15 \mathrm{~cm}^{3}$. A Peltier module on top of the box and a resistive wire wound around the tube are used to cool and heat the system. Each cycle consists of a $240 \mathrm{~min}$ cooling stage followed by a $140 \mathrm{~min}$

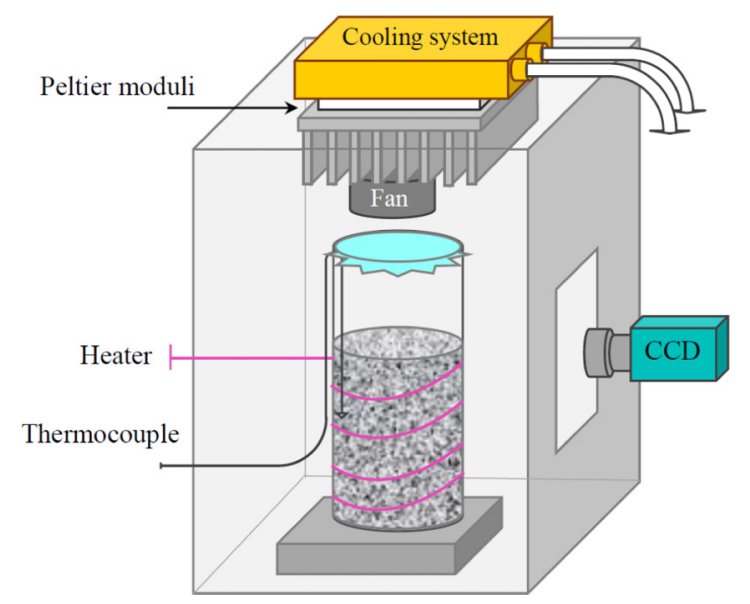

FIG. 1. Sketch of the experimental setup. A Peltier module on top of the box and a resistive wire wound around the tube cool and heat the wet granular pile, respectively. A CCD camera records the position of the granular-air interface. heating stage with the temperature ranging from -6 to $10^{\circ} \mathrm{C}$. The frequency of the temperature variations was chosen small enough to achieve an almost homogeneous heating and freezing of the granular column $\left(4.4 \times 10^{-5} \mathrm{~s}^{-1}\right)$. The thermal cycle was repeated to ensure the saturation of the results. The top granular-air interface is free to move. A CCD camera was used to record the motion of the granular-air interface. The granular packing fraction $\eta$ was determined from the height of the beads column. The volume thermal expansion coefficients of different components of the system (water, grains, container) show the importance of water expansion during freeze-thaw transitions comparing to the other components. The volume thermal expansion coefficient of water varies significantly with the temperature and ranges between -50 and $207 \mu \mathrm{K}^{-1}$ when the temperature goes from 1 to $20^{\circ} \mathrm{C}$. Moreover, during the freezing transition, the water volume increase is around $8 \%$. Therefore, the thermal cycling is expected to modify the granular pile configuration and its packing fraction.

\section{RESULTS}

The time evolution of the packing fraction during one cycle is shown in Fig. 2(a). The liquid volume fraction and the initial packing fraction are fixed at $\omega=0.30$ and $\eta_{0}=0.597$, respectively. A slight contraction of the sample is observed during the decreasing phase of the temperature from 10 to $4{ }^{\circ} \mathrm{C}$, where the water coefficient of thermal expansion decreases to zero [see the temperature curve in Fig. 2(a)]. Then the temperature drops to zero and the liquid-solid phase transition of water begins. During this transition, the temperature inside the pile remains constant near $0^{\circ} \mathrm{C}$. The granular-air
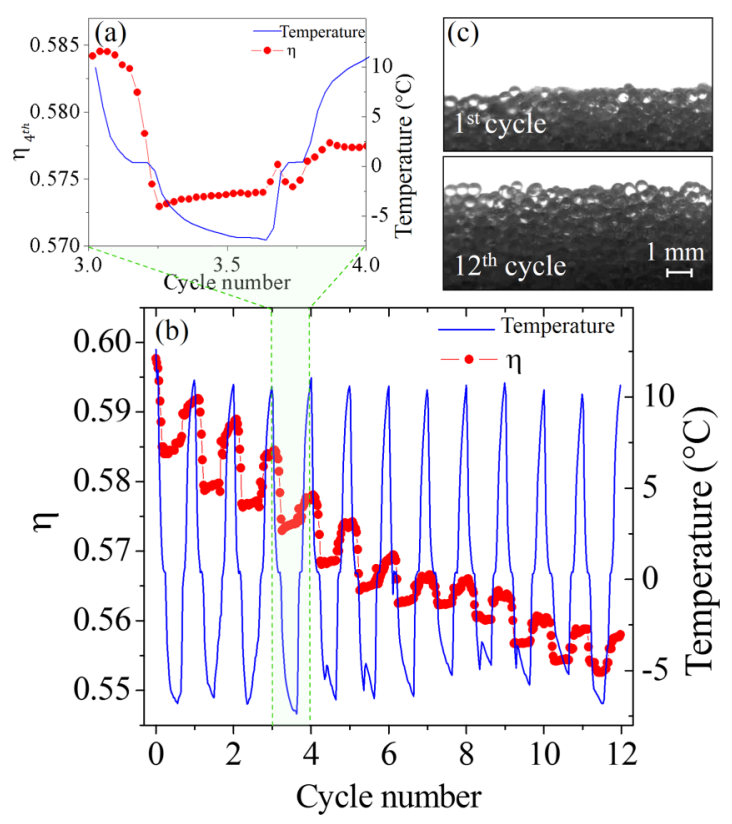

FIG. 2. Evolution of the packing fraction $\eta$ of the granular pile and the temperature inside the pile (a) during one cycle and (b) during 12 cycles. (c) Interface of the granular pile-air after the first cycle and its dilatation after 12 cycles. The initial packing fraction and liquid volume fraction in this experiment are $\eta_{0}=0.597$ and $\omega=0.30$. 


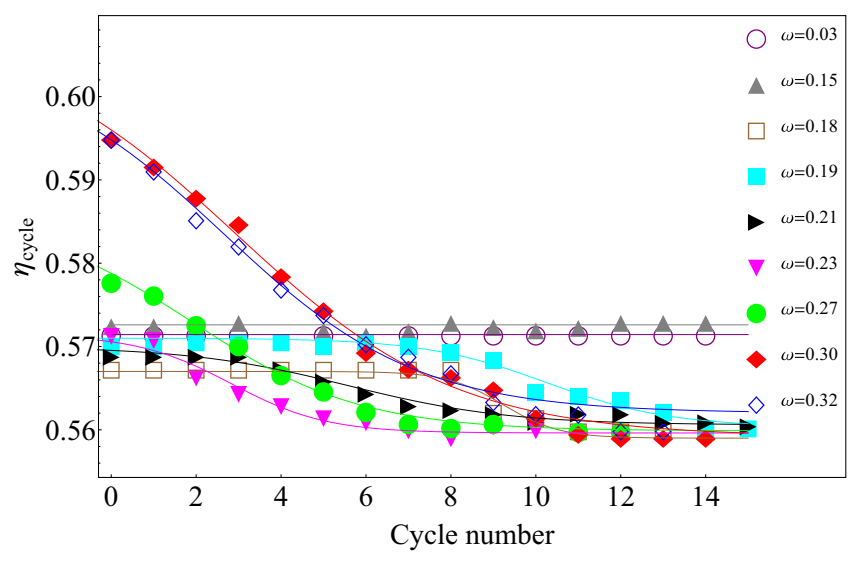

FIG. 3. Evolution of packing fraction $\eta_{\text {cycle }}$ of the piles at the end of each freeze-thaw cycle for different liquid volume fraction $\omega$. The experimental data are fitted (solid lines) with an inverse-exponential function.

interface goes up during the plateau at $0{ }^{\circ} \mathrm{C}$ and the packing fraction $\eta$ decreases. By decreasing the temperature after the plateau at $0{ }^{\circ} \mathrm{C}$, a dilatation of the pile is still observed. Finally, the height of the pile becomes stable. Then the Peltier module is switched off and the heater increases the temperature up to $10{ }^{\circ} \mathrm{C}$. During the heating process, the granular-air interface goes down to reach a plateau after the water melting point.

Figure 2(b) shows a representative time evolution of the packing fraction of a wet pile during 12 thermal cycles. The clear decreasing trend of the packing fraction shows that the mechanism of particle rearrangement is not reversible during the freezing and melting transition. This system is found to experience decompaction to reach a particular value of the packing fraction. This decompaction is particularly visible in the pictures taken before and after the cycling [see Fig. 2(c)]. Nine decompaction curves are presented in Fig. 3 for different liquid volume fractions in the range $0.03 \leqslant \omega \leqslant 0.32$. The packing fraction $\eta_{\text {cycle }}$ is recorded at the end of each freezethaw cycle and plotted as a function of the cycle number $n$. This range of liquid contents covers different regimes of wetness from the creation of the capillary network until the formation of large clusters and finally close to the saturated case. For small liquid volume fraction $\omega=0.03$ up to the intermediate regime $\omega \approx 0.15$, the system tends to stay in its initial state without any volume change during the whole experiment. For liquid volume fraction higher than $\omega \sim 0.15$, the decompaction begins after a number of cycles $n_{\text {on }}$, which is a decreasing function of the liquid volume fraction. For $\omega \gtrsim 0.27$, decompaction begins from the first cycle. Once the decompaction begins, the packing fraction decreases over thermal cycles, and regardless of the liquid volume fraction the systems converge to the random loose packing. The rate of decompaction depends on the liquid content.

Figure 4 shows the dependence of the onset time of dilatation $n_{\text {on }}$ on the liquid volume fraction $\omega$. A liquid volume fraction higher than $\omega \sim 0.15$ was selected for this analysis because no significant dilatation was observed for $\omega \lesssim 0.15$ within the time range of our experiments.

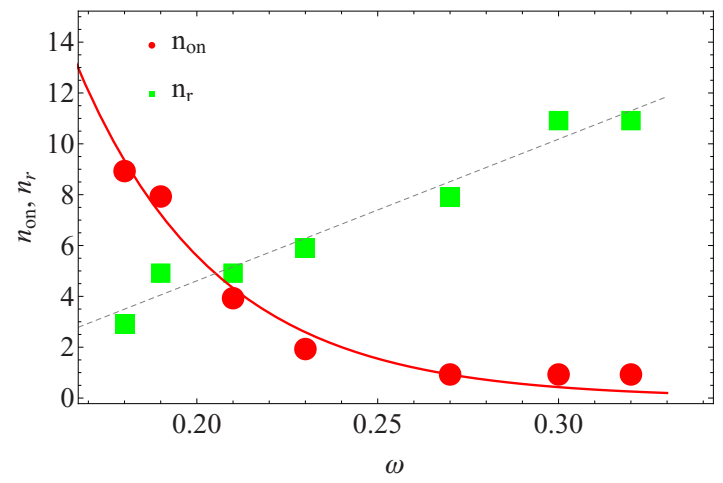

FIG. 4. The onset time (circles) and the relaxation time (squares) of dilation of the granular assemblies as a function of liquid volume fraction $\omega$ to reach the asymptotic value of the packing fraction.

\section{DISCUSSION}

The main observations obtained with the wet granular material column submitted to freeze-thaw cycling are (i) a dilatation of the pile to reach a particular value of the packing fraction $\eta=0.56$, i.e., a value close to our earlier report concerning the saturated case [32], (ii) a minimum value of the liquid volume fraction $\omega \gtrsim 0.15$ to observe this decompaction, and (iii) an onset of the decompaction process after a few cycles, $n_{\text {on }}$. Before this onset, the granular pile is static. While the applied thermal cycling continues, the pile begins to dilate to the asymptotic packing fraction independently of the liquid volume fraction $\omega$ if the condition $\omega \gtrsim 0.15$ is satisfied. Contrary to the saturated case studied previously [32], we never observed any compaction induced by thermal cycling in the present study, and the system tends to reach the random loose packing configuration.

Based on the statistical framework, the evolution of a system of granulates can be evaluated from the fluctuations of the Voronoi cell volumes [40,41]. In our measurements in the presence of liquid, the granulate system experiences a dilatation during freezing. This expansion increases the Voronoi cell volumes. During the melting transition, the mechanism of particles rearrangement is not reversible, and due to this effect the system tends to have a looser configuration. This shows the dissipative behavior of the system during expansion and contraction. So, after several cycles, the non-elastic nature of the clusters movement causes the system to experience decompaction and reach a loose structure.

Another main observation obtained by these series of experiments is the onset of the decompaction process after a few cycles. The dependence of the onset time $n_{\text {on }}$ on the liquid volume fraction and hence on the cohesive force means that the liquid distribution inside the assembly of particles plays an important role in the decompaction dynamics of the system under freeze-thaw cycling. The cohesive forces in the assembly of grains are determined by the curvature of the liquid in the liquid-air interface, and they depend on the surface tension of the liquid. At very low liquid volume fraction $\omega$, the presence of a capillary network makes the contacts between the particles too strong to allow any change in the configuration of the grains. Moreover, the dilatation and the contraction of the liquid during freeze-thaw cycles 
is probably insufficient to move the grains. For higher liquid volume fractions until the Funicular state, the freeze-thaw forcing still does not have any significant macroscopic effect on the piles. Grain motions are observed when the liquid bridges start to form large clusters. These water clusters are expected to experience a dilatation-contraction cycle during the freeze-thaw process, sufficient to modify grain arrangement. Indeed, a careful analysis of the images [see Fig. 2(c)], which shows the evolution of the system during the thermal cycling, illustrates that at $\omega \gtrsim 0.11$, the configuration of the grains changes slightly during the thermal cycling. However, to observe a macroscopic modification of the pile, the liquid volume fraction must be higher than $\omega>0.15$. This means that the effect of Laplace pressure appears in the delay before the onset of dilatation.

Several experimental studies have shown the sensitivity of the mechanical properties of wet granular materials to the liquid volume fraction [20-22]. At low liquid content, the mechanical properties are determined by the liquid bridges between the grains, and those at higher liquid content are determined by the flow of the liquid through the granular assembly. Our study mainly consists of a static or a quasistatic regime for varying liquid content, where the cohesion dominates over other effects of the liquid, such as lubrication and friction. The results at the macroscopic scale for the wet granular pile submitted to freeze-thaw agitation show that despite the microscopic grain rearrangements inside the pile, the packing fraction does not change significantly under the thermal agitation in the range of volume content $\omega \lesssim 0.15$. For the higher liquid volume fraction, the evolution of the system depends on the liquid distribution between the grains.

The evolution of the packing fraction as a function of the cycle number can be well fitted by an inverse-exponential law (see Fig. 3):

$$
\tilde{\eta}(n)=\frac{1}{e^{\left(n-n^{\prime}\right) / n_{r}}+1},
$$

where $\tilde{\eta}$ is the normalized packing fraction, which is defined as $\tilde{\eta}=\left(\eta-\eta_{\infty}\right) /\left(\eta_{0}-\eta_{\infty}\right)$, with $0 \leqslant \tilde{\eta} \leqslant 1$. The parameters $\eta_{0}$ and $\eta_{\infty}$ are the initial and the asymptotic packing fraction, respectively. The parameters $n_{r}$ and $n^{\prime}$ characterize the relaxation time and the process midtime, respectively. We further define the onset time $n_{\text {on }}=n^{\prime}-\frac{n_{r}}{2}$, which represents the time of the onset of the dilatation process. Figure 4 shows the effect of the liquid content on the characteristic parameters $n_{\text {on }}$ and $n_{r}$. Interestingly, the dependence of the onset of dilatation $n_{\text {on }}$ on the liquid volume fraction $\omega$ can be fitted by an exponential function $n_{\text {on }} \propto e^{-\omega / \omega_{0}}$ with $\omega_{0} \approx 0.04$. For the liquid volume fractions in the range of $0.11 \lesssim \omega \lesssim 0.27$, the evolution of the pile is characterized by an onset time along which no macroscopic change is observed in the volume of the system, while microscopic rearrangements are observed above the percolation limit $(\omega \gtrsim 0.11)$. In this range of wetting, microscopic rearrangements act as a stochastic driving force for all the systems, and its stochastic nature is the reason for the presence of an onset time. After the onset time, the behavior of the system is qualitatively similar to $\omega \gtrsim 0.27$, which is characterized by a relaxation time. For the regime with the higher liquid content, the onset time is too short or near zero. This means that the system starts to dilate from the

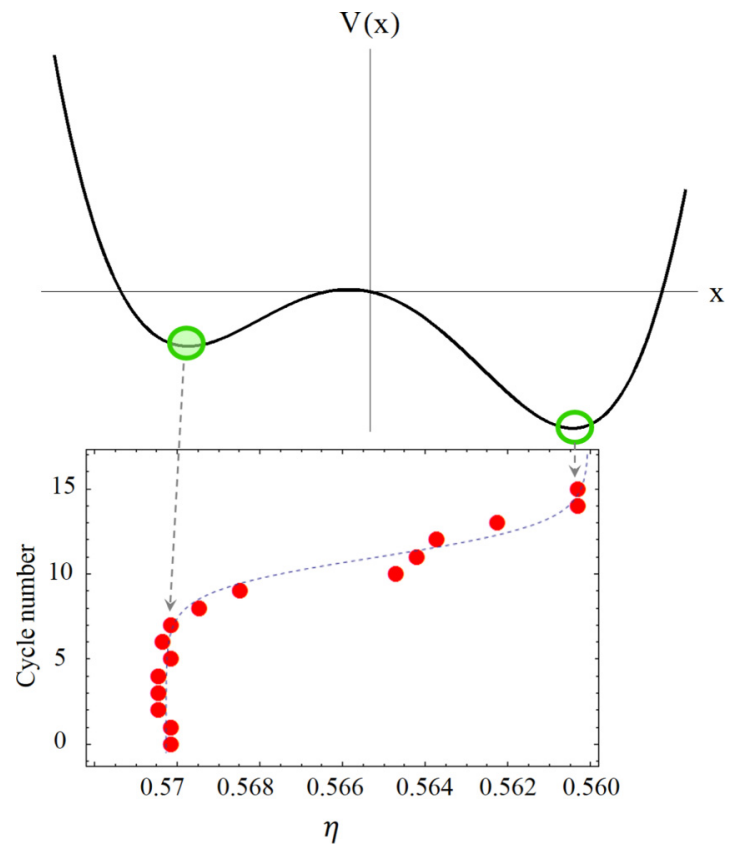

FIG. 5. (a) Schematic representation of a double-well potential due to the thermal cycling. The tilted potential shows the state of the granular pile before and after the decompaction. (b) Experimental result for the liquid volume fraction $\omega=0.19$ with the inverted $x$ axes. The dashed curve is a guide to the eye. These data imply the tendency of the system to stay in a local stable state and reach a global stable state. The onset of decompaction overcomes the barrier related to the cohesive force.

first cycle. Once the transition begins, it takes time $n_{r}$ for the system to relax in the new loose pack state. As is shown in Fig. 4, relaxation time increases almost linearly with liquid content.

The experimental results and the fitted curves presented in Figs. 3 and 4 suggest that the dynamics of the packing fraction of the wet granular materials under the thermal cycling can be modeled by a phenomenological model based on an overdamped particle in a tilted Landau potential under an external force. In this model, the potential can be written as

$$
V(\eta)=\alpha \eta^{4} / 4-\beta \eta^{2} / 2-c \eta,
$$

where $\alpha$ and $\beta$ determine the shape of the Landau potential, and $c$ is the tilt parameter. The parameters are chosen so that with $c=0$ the system has two stable states, and with $c \neq 0$ the potential has one local and one global stable state [see Fig. 5(a)] [42,43]. If the system is initially in the local stable state, external forces can drive the system out of the basin of attraction of the local minimum to bring the system into the other stable state. In this case, the dynamics of the system can be modeled by the equation

$$
\frac{d \eta}{d t}=-\alpha \eta^{3}+\beta \eta+c+\xi(t)
$$

where $\xi(t)$ is a small-amplitude stochastic term. This simplified potential contains the contribution of both gravity and capillary action. The lowest value of the potential in the global stable state corresponding to the lowest packing fraction is due to the contribution of the capillary force. Indeed, with the 
contribution of gravity alone, the lower potential in the global stable state should correspond to a higher packing fraction. The origin of the stochastic term can be the microscopic changes in the configuration of the grains without a macroscopic observable effect. So, the mechanism of dilatation depends on breaking and regeneration of the liquid clusters and rearrangement of the grains [see Fig. 2(c)], which can be characterized by the stochastic parameter $\xi(t)$.

In the model, the barrier energy characterized by $\beta$ is related to the cohesive force and is directly dependent on the liquid volume fraction. The tilt parameter $c$ characterizes the difference between the free energy of the loose and close packed states. Figure 5 is a schematic representation of the relation between the phenomenological model and the transition toward the loose pack configuration seen in our experiments for the liquid volume fraction $\omega=0.19$. We have assumed a positive value for $c$ in our model to identify the transition from close to loose packed states in our experiments.

For very low liquid content less than 0.11 , the movement of the particles under freeze- thaw agitation is very unlikely. This means that the stochastic perturbations are of negligible amplitude, and since in this regime the barrier energy is too high, no macroscopic change is expected. Beyond the percolation limit $\omega \approx 0.11$, microscopic rearrangements are more frequent and they can, in principle, initiate macroscopic dilatation. In the model this is interpreted as a finite amplitude for stochastic perturbations. Still, for the liquid volume fraction in the range $0.11 \lesssim \omega \lesssim 0.15$, we do not observe any dilatation over the course of our experiments. In this regime, the cohesive forces lead to a deep potential well (large $\beta$ ) and make the transition less likely. So the transition time is too long and exceeds the time range of our experiments (15 cycles). Figure 6(a) shows the amplitude of the variation of $\eta$ during each cycle through the time course of all experiments. It shows that the transition to the loose pack state is seen if and only if the system volume undergoes an oscillatory change. The figure also shows that dilatation begins at the same cycle at which oscillations are observed, and during dilatation the amplitude of the oscillations decreases. Also the maximum amplitude of $\eta$ for different volume fractions has been plotted in Fig. 6(b), which shows that the maximum variation increases with volume fraction. These results are in accordance with the argument that the strong cohesive forces act against the macroscopic change in the volume of the wet sand pile.

\section{CONCLUSION}

The packing fraction dynamics of granular materials in different regimes of wetting under freeze-thaw cycling has been investigated. In our previous study dedicated to the saturated case, both compaction and decompaction have been observed depending on the initial packing fraction $\eta_{0}$ [32]. In the present unsaturated case, only decompaction has been observed. The packing fraction dynamics depends strongly on the water content $\omega$ and therefore on the distribution of the liquid inside the assembly of particles. For low liquid content values $\omega \lesssim 0.11$, i.e., less than the percolation threshold, the cohesive forces determined by the curvature of the liquid-air interface are too strong to allow any change in the
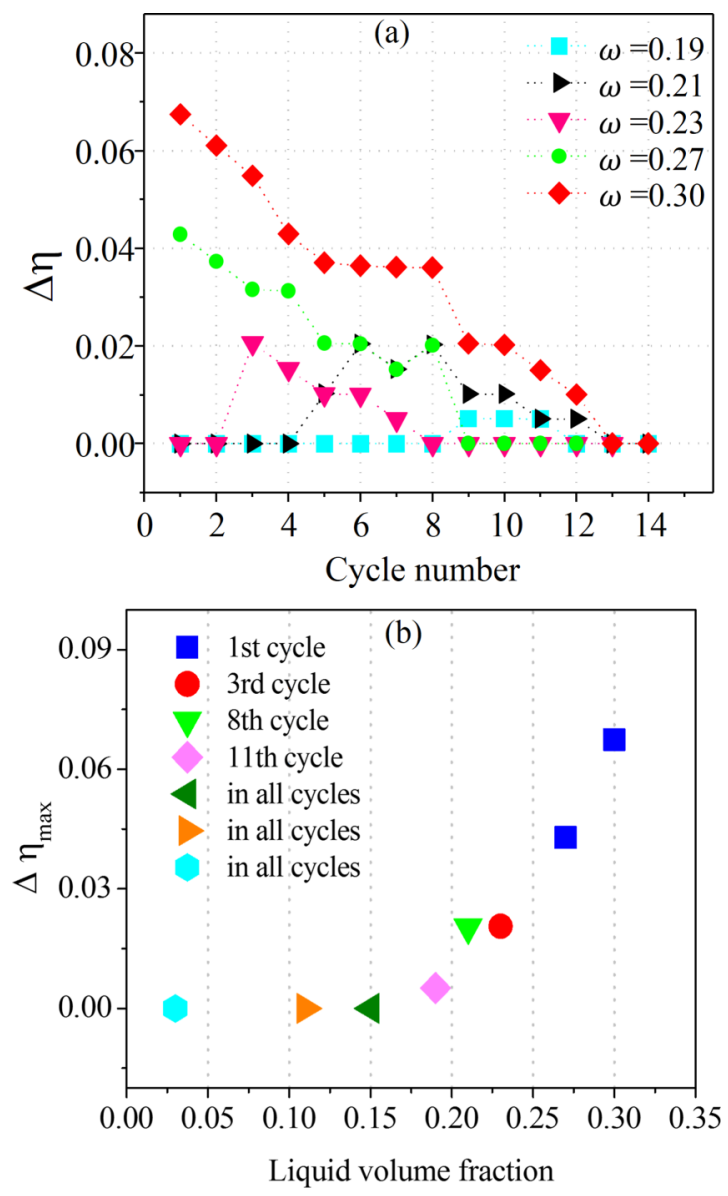

FIG. 6. (a) Amplitude of packing variations during thermal cycles as a function of cycle number for a different regime of wetting, and (b) maximum variation of packing fraction for different liquid volume content.

configuration of the grains under freeze-thaw agitations. Beyond this liquid content, the microscopic rearrangements of the grains occur, which include the breaking and regeneration of the liquid surface in the clusters. These microscopic rearrangements can, in principle, induce a macroscopic dilatation of the pile, and then the packing fraction converges to a particular value of $\eta=0.56$. The evolution of the packing fraction as a function of cycle number is well fitted by an inverse-exponential law. Finally, a delay time for the onset of decompaction has been observed for the liquid content $\omega \lesssim 0.27$. The dependence of the onset time of decompaction $n_{\text {on }}$ on the liquid content $\omega$ is well fitted by a decreasing exponential law.

We have proposed a phenomenological model to identify the physical mechanisms explaining the experimental observed dynamics, based on the Landau potential with a double-well structure. The two wells represent two stable states of the pile observed in the experiments: random loose pack and random close pack states. The main parameters in our model are the height of the barrier energy, which has its root in the cohesive force in the wet pile, and the amplitude of stochastic perturbations, which arise from the microscopic rearrangements within the system. In low volume fractions, the barrier energy is high and the rearrangements are rare 
and no macroscopic change is expected in the system. For higher liquid volume fraction, the Laplace pressure is small, the rearrangements are frequent, and the system begins to dilate at a very early cycle of the experiment. The novel phenomenon is seen in the intermediate levels of the volume fraction $(0.15 \lesssim \omega \lesssim 0.27$ in our experiments) where the dilatation begins after a finite number of cycles. Within the phenomenological framework, we attributed this dynamics to the escape of the system from a potential well due to the presence of stochastic force. Our experiments suggest that the liquid volume fraction is the control parameter that determines the asymptotic behavior of the wet granular material under the freeze-thaw cycling. In our phenomenological model, we have hypothesized that the liquid volume fraction determines the height of the potential barrier and consequently the required energy to move the system toward the loosed packed state.

\section{ACKNOWLEDGMENTS}

This work was financially supported by the FNRS (Grant No. PDR T.0043.14) and by the University of Liège (Starting Grant No. C-13/88). M. Pakpour also acknowledges the support of M. N. Torkamaan Foundation.
[1] J. Bernal and J. Mason, Nature 188, 910 (1960).

[2] M. Hanifpour, N. Francois, V. Robins, A. Kingston, S. M. Vaez Allaei, and M. Saadatfar, Phys. Rev. E 91, 062202 (2015).

[3] C. Radin, J. Stat. Phys. 131, 567 (2008).

[4] Y. Kallus, Soft Matter 12, 4123 (2016).

[5] M. Jerkins, M. Schröter, H. L. Swinney, T. J. Senden, M. Saadatfar, and T. Aste, Phys. Rev. Lett. 101, 018301 (2008).

[6] B. M. Guy, M. Hermes, and W. C. K. Poon, Phys. Rev. Lett. 115, 088304 (2015).

[7] I. Zuriguel, A. Garcimartín, D. Maza, L. A. Pugnaloni, and J. M. Pastor, Phys. Rev. E 71, 051303 (2005).

[8] N. Xu, V. Vitelli, M. Wyart, A. J. Liu, and S. R. Nagel, Phys. Rev. Lett. 102, 038001 (2009).

[9] R. Lespiat, S. Cohen-Addad, and R. Höhler, Phys. Rev. Lett. 106, 148302 (2011).

[10] A. Samadani and A. Kudrolli, Phys. Rev. Lett. 85, 5102 (2000).

[11] J. Ottino and D. Khakhar, Annu. Rev. Fluid Mech. 32, 55 (2000).

[12] J. Duran, E. Kolb, and L. Vanel, Phys. Rev. E 58, 805 (1998).

[13] P. Richard, M. Nicodemi, R. Delannay, P. Ribiere, and D. Bideau, Nat. Mater. 4, 121 (2005).

[14] G. Lumay and N. Vandewalle, Phys. Rev. Lett. 95, 028002 (2005).

[15] M. J. Blunt, Current Opin. Colloid Interface Sci. 6, 197 (2001).

[16] J. E. Fiscina, M. Pakpour, A. Fall, N. Vandewalle, C. Wagner, and D. Bonn, Phys. Rev. E 86, 020103 (2012).

[17] P. C. Møller and D. Bonn, Europhys. Lett. 80, 38002 (2007).

[18] M. Pakpour, M. Habibi, P. Møller, and D. Bonn, Sci. Rep. 2, 549 (2012).

[19] A. Kudrolli, Nat. Mater. 7, 174 (2008).

[20] M. Scheel, R. Seemann, M. Brinkmann, M. Di Michiel, A. Sheppard, B. Breidenbach, and S. Herminghaus, Nat. Mater. 7, 189 (2008).

[21] A. Fall, B. Weber, M. Pakpour, N. Lenoir, N. Shahidzadeh, J. Fiscina, C. Wagner, and D. Bonn, Phys. Rev. Lett. 112, 175502 (2014).

[22] Z. Fournier, D. Geromichalos, S. Herminghaus, M. Kohonen, F. Mugele, M. Scheel, M. Schulz, B. Schulz, C. Schier, R. Seemann et al., J. Phys.: Condens. Matter 17, S477 (2005).
[23] S. Herminghaus, Adv. Phys. 54, 221 (2005).

[24] N. Mitarai and F. Nori, Adv. Phys. 55, 1 (2006).

[25] J. E. Fiscina, G. Lumay, F. Ludewig, and N. Vandewalle, Phys. Rev. Lett. 105, 048001 (2010).

[26] J. B. Knight, C. G. Fandrich, C. N. Lau, H. M. Jaeger, and S. R. Nagel, Phys. Rev. E 51, 3957 (1995).

[27] O. Pouliquen, M. Belzons, and M. Nicolas, Phys. Rev. Lett. 91, 014301 (2003).

[28] R. Jackson, The Dynamics of Fluidized Particles (Cambridge University Press, Cambridge, 2000).

[29] M. Schröter, D. I. Goldman, and H. L. Swinney, Phys. Rev. E 71, 030301 (2005).

[30] K. Chen, J. Cole, C. Conger, J. Draskovic, M. Lohr, K. Klein, T. Scheidemantel, and P. Schiffer, Nature (London) 442, 257 (2006).

[31] B. Blanc and J.-C. Géminard, Phys. Rev. E 88, 022201 (2013).

[32] F. Ludewig, N. Vandewalle, S. Dorbolo, M. Pakpour, and G. Lumay, Phys. Rev. E 92, 010202 (2015).

[33] R. W. Style, S. S. L. Peppin, A. C. F. Cocks, and J. S. Wettlaufer, Phys. Rev. E 84, 041402 (2011).

[34] T. Saruya, K. Kurita, and A. W. Rempel, Phys. Rev. E 87, 032404 (2013).

[35] J. E. Sawicka, A. Robador, C. Hubert, B. B. Jørgensen, and V. Brüchert, ISME J. 4, 585 (2010).

[36] P. Rognon and I. Einav, Phys. Rev. Lett. 105, 218301 (2010).

[37] E. Kolstrup and T. Thyrsted, Geomorphology 129, 361 (2011).

[38] P. Viklander and D. Eigenbrod, Cold Regions Sci. Technol. 31, 151 (2000).

[39] B. J. Thomson and J. W. Head III, J. Geophys. Res. 106, 23 (2001).

[40] C. Xia, J. Li, Y. Cao, B. Kou, X. Xiao, K. Fezzaa, T. Xiao, and Y. Wang, Nat. Commun. 6, 8409 (2015).

[41] T. Aste and T. Di Matteo, Eur. Phys. J. B 64, 511 (2008).

[42] S. H. Strogatz, Nonlinear Dynamics and Chaos: With Applications to Physics, Biology, Chemistry, and Engineering (CRC Press, Boca Raton, Florida, 2018).

[43] A. Valizadeh, K. Jahanbani, and M. R. Kolahchi, Phys. Rev. A 81, 023616 (2010). 